\title{
The Effect of Collusion Games on the Profit of Primary Networks and Secondary Heterogeneous Cognitive Radio Networks with Femtocells
}

\author{
Yousef N. Shnaiwer \\ Electrical Engineering Department, KFUPM \\ Dhahran, Saudi Arabia \\ Email: yousefshnaiwer@gmail.com
}

\author{
Salam A. Zummo Senior Member, IEEE \\ Electrical Engineering Department, KFUPM \\ Dhahran, Saudi Arabia \\ Email: zummo@kfupm.edu.sa
}

\begin{abstract}
This paper studies the effect of the different pricing games played by the primary user (PU) operators on the profit of both the PU networks and the cognitive base station (CBS). We show that the price achieved by the PU operators in the collusion game (called the Pareto point) is the maximum price that can be achieved in the competitive game when the substitutability parameter is higher than zero. However, for negative values of the substitutability parameter, the Pareto point represents the minimum price that can be offered by the PU operators. Moreover, the effect of the collusion game on the profit of the CBS has also been investigated, and the simulations show that the worst case for the CBS in terms of profit is not associated with the highest price that can be offered by the PU networks.

Index Terms-Femtocell, cognitive radio, Nash equilibrium, Pareto point, collusion games, Stackelberg games.
\end{abstract}

\section{INTRODUCTION}

Cognitive radio networks have been proposed as a solution to enhance spectrum and energy efficiencies by extending the bandwidth choices of the radio and thus lessen the need for increasing the power to satisfy network capacity requirements [1]. The concept of cognitive radio hinges on the existence of primary users (PU's) and secondary users (SU's). The PU's are authorized to utilize the spectrum whereas the SU's are not. So the SU's should sense the spectrum and check for spectrum holes in a trial to maximize its quality of service (QoS) while minimizing interference with the PU's [2]. This is the general scenario for cognitive radio networks.

Several scenarios for the implementation of cognitive radios have been investigated in the research community. One of these scenarios is the one studied in $[3,4]$ in which there are different operators serving their own users and competing (or colluding) to serve the secondary users to maximize their profits.

Another possible scenario for cognitive radio networks is the so called trading mechanism [5]. In this scenario, the PU networks have surplus spectrum (i.e. the PU networks are not using the whole spectrum to serve their users) which can be sold to the SU networks to gain more revenue for the PU operators. Of course, this will result in service degradation for the PU networks. The existence of several PU networks in the same area of the SU network will trigger price competition among the PU networks in an attempt to sell more spectrum to the SU network. A cognitive base station (CBS) is supposed to purchase spectrum from the PU networks.

In [6], the authors presented a game-theoretical model for the price competition between the PU networks to sell spectrum to a two-tier cognitive radio network (i.e. the CBS with its users, and the femtocell base stations (FBS's) with their users). The price competition game has been modelled as a three-stage Stackelberg game. Stackelberg game is a multistage game in which two types of players are involved, namely, leaders and followers. The followers takes their moves based on the moves of the leaders[6]. Here, the leaders are the PU networks, and the followers are the CBS and the FBS's. Each of these players is trying to maximize its profit by considering the moves of the other players. The price competition between the PU networks is just the first stage of the Stackelberg game, and the Nash equilibrium is the solution of that stage. The authors presented an algorithm to find the Stackelberg equilibrium solution which depends basically on finding the solution of each stage and using this solution as an input to the next stage. However, the authors in [6] did not provide any stability analysis for their algorithm. Stability is necessary for any dynamic algorithm to ensure that the Nash equilibrium can be reached in the steady state [5]. Furthermore, the effect of the collusion established (and maintained) by the PU networks on their profits and the profit of the CBS has not been studied for this scenario.

In this paper, we compare the collusion game to the pricecompetition game for the scenario presented in [6] in terms of the PU's and the CBS profit. Before doing so, we perform the stability analysis for the Stackelberg algorithm and find the conditions for this algorithm to be stable for the general case of $L$ PU networks. The optimum step size for the algorithm in terms of the speed of convergence is also determined. We also study the effect of the substitutability parameter and the energy efficiency of the macrocell SU's (MSU's) and the FBS's on the resultant Nash and Pareto prices. Furthermore, we prove that Pareto price is not the price that will result in the highest profit for PU's as argued in [5].

The remainder of this paper is organized as follows. In Section II, the system model is illustrated. The stability 
analysis is presented in Section III. The equations of the Pareto price and the Nash equilibrium price are derived, compared, and the effect of some parameters on both of them is discussed in Section IV. The simulation results and discussions are provided in Section V, and Section VI contains the conclusion and recommendations.

\section{System Model}

As shown in Fig. 1, there exist $L$ PU networks, and $K$ SU's ( $K_{1}$ FBS's and $K_{2}$ MSU's, where $K_{1}+K_{2}=K$ ) in the SU network. Each PU network offers a price $c_{l}$ to the CBS and each user sends its energy efficiency $\eta_{k}$ to the CBS. Each FBS serves one femtocell SU (FSU). The energy efficiency of each user (MSU or FSU) is defined as [6]

$$
\eta_{k}=\frac{\log _{2}\left(1+\frac{h_{k}^{2} p_{k}}{\sigma^{2}}\right)}{p_{a}+p_{k}},
$$

where $p_{k}$ and $h_{k}$ are the energy-efficient power allocation of user $k$ and the channel gain from that user (MSU or FSU) to its base station (CBS or FBS), respectively, $p_{a}$ is the additional power consumption due to transmission, and $\sigma^{2}$ is the noise power assuming an additive white Gaussian noise (AWGN) with zero mean. It is assumed here that the price information sent by each PU is given per unit bandwidth. So the CBS knows what the maximum spectrum bandwidth that can be purchased from PU networks is, and it will inform the FBS's to use that bandwidth to calculate the energy efficiency of the transmission of their FSU's. Next, the CBS will allocate the spectrum band with the lowest price to the user with the highest energy efficiency to maximize its revenue. This is done by setting the spectrum allocation index $x_{l k}$ of that user to 1 . It is important to note here that the energy-efficient power allocation is the transmit power of each user (MSU or FSU) that maximizes its energy efficiency.

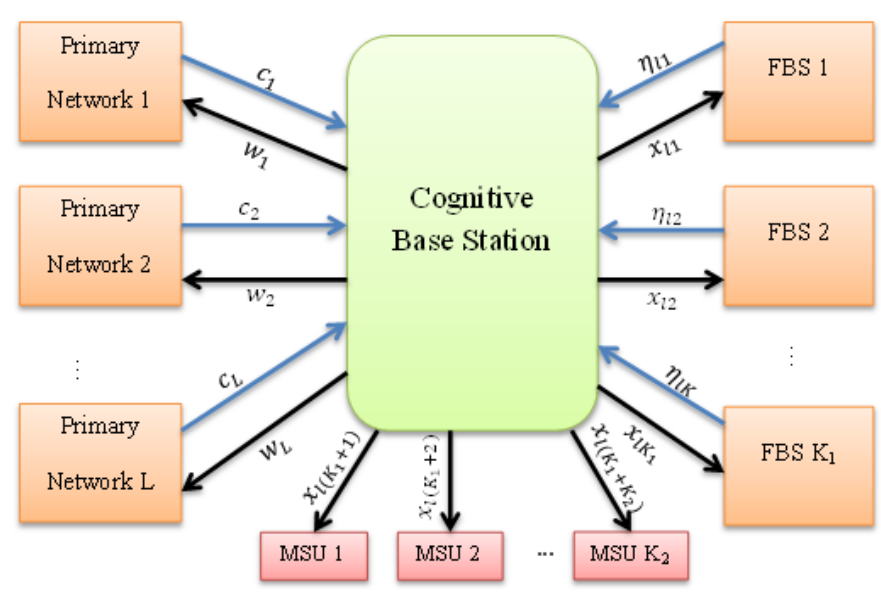

Fig. 1: The general system model.

We assume a static environment in which the channel conditions of users do not change over the trading period. The CBS purchases the spectrum bands that maximize its revenue from the spectrum offered by $L$ PU operators. Then, the CBS uses that spectrum to serve the MSU's and FBS's according to their energy efficiencies to maximize its profit (i.e. the cheapest spectrum band is allocated to the MSU/FBS with the highest energy efficiency).

Each spectrum band purchased by the CBS from a PU network is allocated only to one MSU/FBS. Each FBS serves only one user at each time slot. We assume that the number of SU's that can access the CBS at each time slot is equal to the number of bands offered by the PU networks $(K=L)$. The FBS's are connected to the CBS via broadband connection (e.g. digital subscriber line (DSL)).

\section{Stability Anlysis FOR the Stackelberg ALGORIOTHM}

In this section, the stability of the Stackelberg algorithm proposed in [6] is analyzed, the stability conditions are derived, and the optimal step size is determined. First of all, we start this section by revising the Stackelberg algorithm and presenting the equations upon which the work in the rest of this paper will be based.

\section{A. The Stackelberg Algorithm}

As mentioned in the previous section, the algorithm starts with the users sending their energy efficiencies and the PU networks sending their offers (prices/bandwidth) to the CBS. The CBS determines the spectrum demand from each PU network that will maximize its profit. The CBS profit $\left(P_{C B S}\right)$ and the spectrum demand $(w)$ can be calculated as follows $[5,6]$

$$
P_{C B S}(w)=\sum_{l=1}^{L} w_{l} K_{\eta l}-\frac{1}{2}\left\{\sum_{l=1}^{L} w_{l}^{2}+2 v \sum_{q \neq l} w_{l} w_{q}\right\}-\sum_{l=1}^{L} c_{l} w_{l}
$$

$$
w_{l}=\frac{N\left(K_{\eta l}-c_{l}\right)-v \sum_{q \neq l}\left(K_{\eta q}-c_{q}\right)}{M},
$$

where $K_{\eta l}=\sum_{k=1}^{K} \zeta_{k} x_{l k} \eta_{k}$ is the revenue of the CBS from spectrum band $l, N=v(L-2)+1$, and $M=(1-v)(v(L-$ $1)+1) ; \zeta_{k}$ is the cost paid by the $k$ 'th user (MSU/FBS) to the CBS, $x_{l k}$ is the spectrum allocation index which when equal to 1 , indicates that the spectrum band purchased from PU $l$ is allocated to the user $k ; v \in[-1,1]$ is the substitutability parameter. When $v$ is larger than 0 , this means the CBS can switch between the spectrum bands of different PU networks; when it is equal to 0 , this means that the CBS can not switch among the spectrum because of the large penalty set by the PU operator on doing so [5]. When $v$ is lower than 0, this means the purchased band is complementary (i.e. the CBS should purchase another band to make use of this band, for example when the CBS purchases the downlink band, it needs to purchase the uplink band also [5]).

So now, the CBS has determined its spectrum demand from each PU network. Using the spectrum demand, each PU can find its profit and the amount by which the offered price 
should be modified which is simply the derivative of the profit multiplied by some weighting factor. The profit of each PU and its derivative are determined as in Eq.'s (4) and (5) respectively [6]

$$
\begin{aligned}
P_{P U_{l}}(c)= & a \eta_{s l}\left(B_{l}-w_{l}\right)+c_{l} w_{l} \\
= & a \eta_{s l}\left\{B_{l}-\frac{N\left(K_{\eta l}-c_{l}\right)-v \sum_{q \neq l}\left(K_{\eta q}-c_{q}\right)}{M}\right\} \\
& +c_{l}\left\{\frac{N\left(K_{\eta l}-c_{l}\right)-v \sum_{q \neq l}\left(K_{\eta q}-c_{q}\right)}{M}\right\}
\end{aligned}
$$

$$
\frac{\partial P_{P U_{l}}(c)}{\partial c_{l}}=\frac{N\left(a \eta_{s l}+K_{\eta l}-2 c_{l}\right)-v \sum_{q \neq l}\left(K_{\eta q}-c_{q}\right)}{M}
$$

where $\eta_{s l}$ is the spectrum efficiency of the l'th PU, and $a$ is the cost paid by the PU's to the PU network, and $B_{l}$ is the total bandwidth licensed to the PU network $l$.

The self-mapping function proposed in [6] to modify the PU's prices is as follows

$$
c_{l}(t+1)=c_{l}(t)+\mu \frac{\partial P_{P U_{l}}(c)}{\partial c_{l}},
$$

where $\mu$ denotes the iterative step size of the price. The algorithm will terminate when the difference between prices from two consecutive iterations is lower than a certain value. The closer this values is to 0 , the lower the error in the resultant optimum price.

\section{B. Stability of the Self-Mapping Function}

In order to examine the stability of the algorithm, it is necessary to check whether the self-mapping function converges or not. This can be done by finding the Jacobean matrix of the self-mapping function and determining its eigenvalues. If all of the eigenvalues of the Jacobean matrix are inside the unit circle, this means that the self-mapping function is stable and thus, the algorithm is stable [5]. The Jacobean matrix of the self-mapping function for $L$ PU networks is defined as follows

$$
J=\left(\begin{array}{cccc}
\frac{\partial c_{1}(t+1)}{\partial c_{1}(t)} & \frac{\partial c_{1}(t+1)}{\partial c_{2}(t)} & \cdots & \frac{\partial c_{1}(t+1)}{\partial c_{L}(t)} \\
\frac{\partial c_{2}(t+1)}{\partial c_{1}(t)} & \frac{\partial c_{2}(t+1)}{\partial c_{2}(t)} & \ldots & \frac{\partial c_{2}(t+1)}{\partial c_{L}(t)} \\
\vdots & \vdots & \ddots & \vdots \\
\frac{\partial c_{L}(t+1)}{\partial c_{1}(t)} & \frac{\partial c_{L}(t+1)}{\partial c_{2}(t)} & \cdots & \frac{\partial c_{L}(t+1)}{\partial c_{L}(t)}
\end{array}\right) .
$$

By differentiating all the self-mapping functions in (6) with respect to all prices $\left\{c_{l}\right\}_{l=1}^{L}$, the resultant Jacobean matrix can be written as

$$
J=\left(\begin{array}{cccc}
1-\frac{2 \mu N}{M} & \frac{\mu v}{M} & \cdots & \frac{\mu v}{M} \\
\frac{\mu v}{M} & 1-\frac{2 \mu N}{M} & \cdots & \frac{\mu v}{M} \\
\vdots & \vdots & \ddots & \vdots \\
\frac{\mu v}{M} & \frac{\mu v}{M} & \cdots & 1-\frac{2 \mu N}{M}
\end{array}\right) .
$$

This matrix is a special case of the Touplitz circulant matrix [7], where the values around the diagonal are identical. The eigenvalues of an $L \times L$ circulant matrix can be found as follows [8]

$$
\lambda_{m}=\rho_{0}+\rho_{L-1} \omega_{m}+\rho_{L-2} \omega_{m}^{2}+\ldots+\rho_{1} \omega_{m}^{L-1},
$$

where $m=0, \ldots, L-1, \omega_{m}=\exp \left(\frac{j 2 \pi m}{L}\right), \rho_{0}$ is the value in the diagonal, and $\rho_{L-1}, \ldots, \rho_{1}$ are the values around the diagonal which are identical in our case . Let $\rho$ denotes the coefficient outside the diagonal then, (9) becomes

$$
\lambda_{m}=\rho_{0}+\rho \sum_{n=1}^{L-1} \exp \left(\frac{j 2 \pi m n}{L}\right) .
$$

The summation in Eq. (10) can be simplified further

$$
\begin{aligned}
\sum_{n=1}^{L-1} \exp \left(\frac{j 2 \pi m n}{L}\right) & =-1+\sum_{n=0}^{L-1} \exp \left(\frac{j 2 \pi m n}{L}\right) \\
& = \begin{cases}L-1, & m=0 ; \\
-1+\frac{1-\exp (j 2 \pi m)}{1-\exp \left(\frac{j 2 \pi m}{L}\right)}, & m \neq 0 .\end{cases} \\
& = \begin{cases}L-1, & m=0 ; \\
-1, & m \neq 0 .\end{cases}
\end{aligned}
$$

Now, we have $\lambda_{0}=\rho_{0}+\rho[L-1]$ and $L-1$ eigenvalues $\lambda_{m}=$ $\rho_{0}-\rho$. Substituting the values of $\rho_{0}$ and $\rho$ and simplifying, the eigenvalues become

$$
\begin{aligned}
& \lambda_{0}=1-\mu\left[\frac{2 N+v(L-1)}{M}\right]=1-\mu\left[\frac{v(L-3)+2}{M}\right], \\
& \lambda_{m}=1-\mu\left[\frac{2 N-v}{M}\right], m=1 \ldots L-1 .
\end{aligned}
$$

To find the stability conditions, the inequality $|\lambda|<1$ should be solved for both terms in (12). By solving the inequality, the stability conditions are as follows

$$
0<\mu<\min \left(\frac{2 M}{2 N-v}, \frac{2 M}{N-v+1}\right) .
$$

Fig. 2 shows $v$ against the upper limit in (13). We can notice that the stability range of the Stackelberg algorithm tends to shrink when $v$ is increasing with the increase of $L$. This means that maintaining a low value of $v$ will give the PU networks a wider range of iterative step sizes to choose from while staying in the stable region.

Lemma 1: The optimal step size for the Stackelberg algorithm in terms of convergence speed is $\mu=\frac{M}{2 N}$.

Proof: We start by taking two consecutive prices, $c_{1}$ and $c_{2}=c_{1}+\mu \frac{\partial P_{P U_{l}}\left(c_{1}\right)}{\partial c_{l}}$. Using (5) and substituting $c_{2}$, the derivative of the second price can be expressed as in (14) at the top of the next page. 


$$
\begin{aligned}
\frac{\partial P_{P U_{l}}\left(c_{2}\right)}{\partial c_{l}} & \left.=\frac{N\left(a \eta_{s l}+K_{\eta l}-2\left[c_{1}+\mu \frac{\partial P_{P U_{l}}\left(c_{1}\right)}{\partial c_{l}}\right]\right)-v \sum_{q \neq l}\left(K_{\eta q}-c_{q}\right)}{M}\right] \\
& =\frac{N\left(a \eta_{s l}+K_{\eta l}-2\left[c_{1}+\mu\left[\frac{N\left(a \eta_{s l}+K_{\eta l}-2 c_{1}\right)-v \sum_{q \neq l}\left(K_{\eta q}-c_{q}\right)}{M}\right]\right)-v \sum_{q \neq l}\left(K_{\eta q}-c_{q}\right)\right.}{M}
\end{aligned}
$$

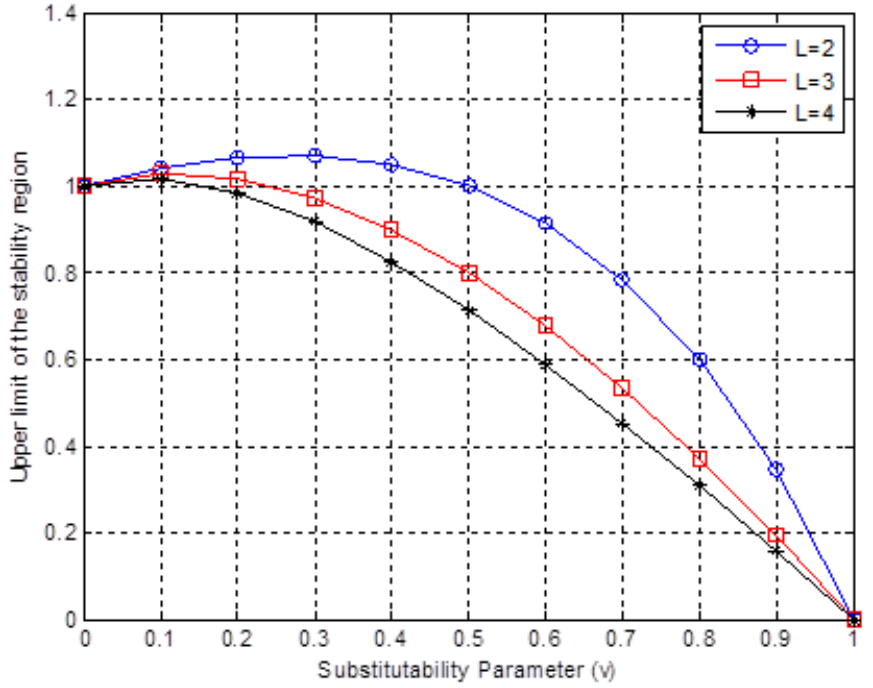

Fig. 2: The substitutabilty parameter (v) against the upper limit of Eq. (13) for different values of $L$.

To find $\mu$, the equation to be solved is given by

$$
\frac{\partial P_{P U_{l}}\left(c_{2}\right)}{\partial c_{l}}=\epsilon,
$$

where $\epsilon$ is a small number. Solving (15) for $\mu$ and observing as $\epsilon \rightarrow 0$, we find that $\mu \rightarrow \frac{M}{2 N}$. Reaching zero at this step indicates that the prices has reached any other value of $\epsilon>0$ before this step. This means that at any other value of $\epsilon$ higher than zero, the lowest number of steps to reach $\epsilon$ is achievable at $\mu=\frac{M}{2 N}$. Hence, the optimal iterative step size is

$$
\mu=\frac{M}{2 N} \text {. }
$$

\section{The Collusion Game and the Maximum Price FOR PU NETWORKS}

In this section, the equations for Nash equilibrium and Pareto point are presented, and we compare between the two equations in terms of the effect of the substitutability parameter and energy efficiency on each of them.

\section{A. Nash and Pareto Prices}

The Nash price is the price that maximizes the profit of each PU network. Therefore, to find the Nash price, all what we need is to solve $\frac{\partial P_{P U_{l}}(c)}{\partial c_{l}}=0$ for each PU network. At the equilibrium, all the prices are almost the same. Assuming all the users has the same energy efficiency, and substituting $c_{l}$ instead of $c_{q}$ in (5), the equation to be solved to find the Nash price becomes

$$
N\left(a \eta_{s l}+K_{\eta l}-2 c_{l}\right)-v(L-1) K_{\eta l}+v(L-1) c_{l}=0 .
$$

Solving (17) for $c_{l}$, the Nash price for any number $L$ of PUs can be written as

$$
c_{N a s h}=\frac{N\left(a \eta_{s l}+K_{\eta l}(1-v)\right)}{2+v(L-3)} .
$$

An important point to notice here is that the Nash price decreases with the increase of the number of PU networks. The rationale behind this decrease in price is that when the number of PU networks increases, the competition among them will increase because the spectrum demand will be distributed over a larger number of operators. So each PU network now needs to take into account a larger number of other moves, and this will cause the price to be more conservative with the increase of $L$.

For the Pareto case, the sum of the profits of all PU operators should be maximized. Hence, we need to solve $\frac{\partial \sum_{l=1}^{L} P_{P U_{l}}(c)}{\partial c_{l}}=0$. Again, at the Pareto point, all the prices are the same, so after differentiating the sum, we substitute $c_{l}$ instead of $c_{q}$. After differentiating and solving for $c_{l}$, the Pareto price is written as

$$
c_{\text {Pareto }}=\frac{a \eta_{s l}+K_{\eta l}}{2} .
$$

Note that the Pareto price does not depend on the substitutability parameter or the number of PU networks; rather, it depends on the energy efficiency of the secondary users (which means more spectrum demand is expected) and the spectrum efficiency of the transmission of primary users (which means more revenue is expected from primary users). The substitutability parameter has no effect on the Pareto point, but it helps the PU operators to reach the Pareto point in the competition game by decreasing it (when it is positive). On the other hand, the energy efficiency changes the Pareto point. That is, when the energy efficiency of the SU's increases, they are expected to use the spectrum more extensively. Thus, the spectrum demand of the CBS is expected to increase and hence the increase in the maximum price that can be offered by the PU operators. 


\section{B. The Maximum Pricing (Pareto Superior)}

Before discussing the maximum achievable price for PU networks, it should be noticed here that we are interested in the positive values of (18) since it is a price (i.e. $L \geq 2$ for $v \in[-1,1])$. To understand the relation between Nash and Pareto prices, we take the difference between Eq.'s (18) and (19) and investigate when this difference is higher than zero

$$
c_{\text {Nash }}-c_{\text {Pareto }}>0 .
$$

Substituting and simplifying, the inequality in (20) becomes

$$
v(1-2 L) K_{\eta l}+v(L-1) a \eta_{s l}>0 .
$$

When the inequality in (21) is satisfied, the Nash price will be higher than the Pareto price. At first, we need to point out that the condition $c_{l}>a \eta_{s l}$ should be satisfied in order for the PU network to prefer selling the spectrum to the CBS [6]. But $K_{\eta l}$ should be higher than $c_{l}$ for the CBS to prefer buying the spectrum from the PU network. Hence, $K_{\eta l}>a \eta_{s l}$. The term $1-2 L$ is absolutely larger than $L-1$ but is negative for $L \geq 2$. This means that when the substitutability parameter $v$ is higher than zero, the Pareto price is always higher than the Nash price. On the contrary, when $v$ is lower than zero, the Nash price is always higher than the Pareto price.

Based on the aforementioned discussion, the Nash price is always higher than Pareto price when $v$ is negative, but what does it mean when $v$ is negative? To understand, we need to know more about competitive pricing. The type of game played here is called Bertrand game [9], in which the firm (PU the operator in our case) determines the price and the user (the CBS in our case) determines the spectrum demand. Such a game is usually preferred by the firms when the good is a complement [10] which is, as illustrated at the beginning of Section III, the case of $v$ being negative. To demonstrate the meaning of complement spectrum, we re-evoke the example given in [5] with some modifications to make it suitable for our scenario. When the CBS needs two spectrum bands, one for the uplink and the other one for the downlink, each one of these bands is considered a complement (i.e. it can not be used alone). A practical example of this case is LTE-FDD [11] which needs two separate bands for the uplink and downlink channels. On the other hand, LTE-TDD can use the same band for the two channels.

\section{Simulation Results AND Discussion}

In the simulations, the number of PU networks is 2, the spectral efficiency of PU transmission $=2 \mathrm{bits} / \mathrm{s} / \mathrm{Hz}$, the energy efficiency of SU transmission is $22.1444 \mathrm{bits} / \mathrm{Hz} / \mathrm{Joule}$, the additional circuit power consumption is $0.1 \mathrm{~W}$, and the cost paid by the PU to its operator and by the SU to the CBS is set to 1 . It is assumed here that the number of users is equal to the number of the PU networks (i.e., $L=K$ ).

\section{A. Nash and Pareto Prices}

The profit of PU networks versus the Nash price is shown in Fig. 3 for different (postitive) values of $v$. The values pointed by the circles have been found using (19). It is obvious that the Pareto point achieves the highest profit for the PU operators.

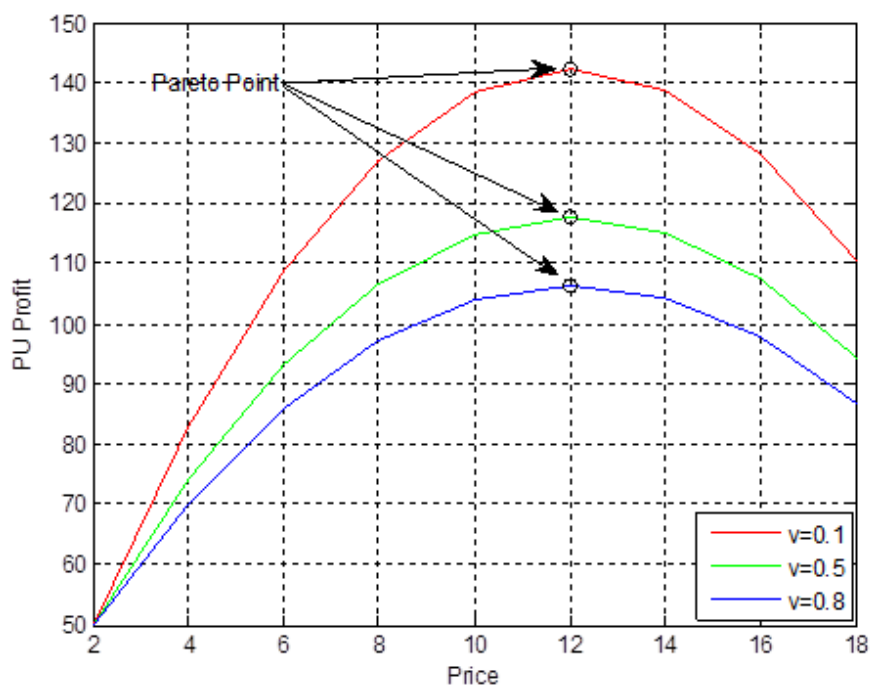

Fig. 3: The profit of PUs versus Nash price for different values of the substitutability parameter $(v)$.

The approach of the Nash price to the Pareto point with the decrease of the (positive) value of $v$ is shown in Fig. 4. Here, the Pareto point is the highest price, while for negative values of $v$, Pareto is the lowest price that can be offered by the PU's. This is shown in Fig. 5.

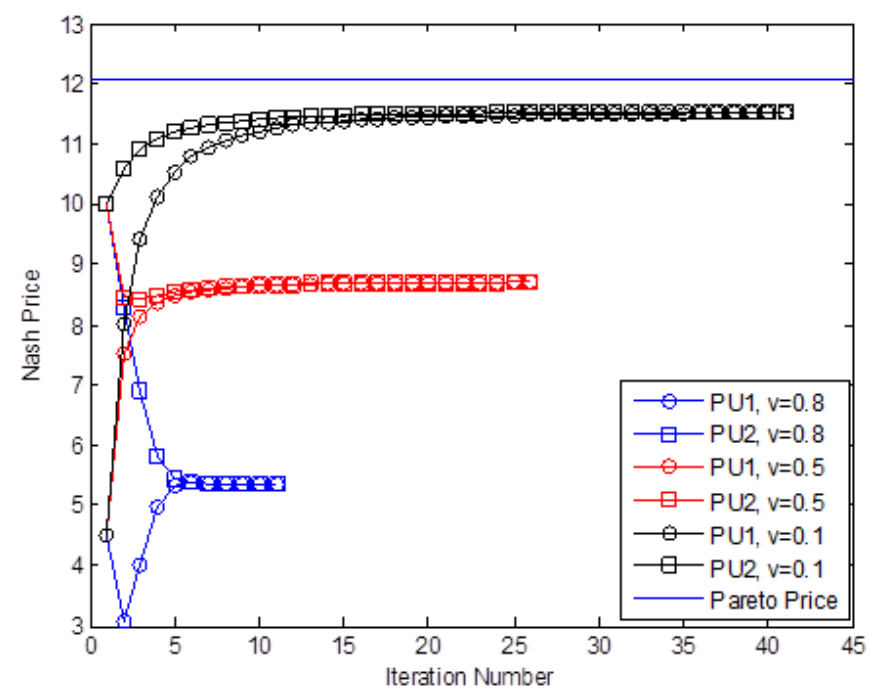

Fig. 4: The convergence of Nash price to Pareto price with the decrease of the (positive) value of $v$.

\section{B. CBS Profit}

Fig. 6 shows the maximum profit (per spectrum demand) that can be achieved by the CBS against the substitutability parameter $v$ for different numbers of the PU networks. The first note is that the minimum (maximum) profit the CBS can achieve depends on the number of PU networks. This is the worst case for the CBS in terms of profit. The important note 


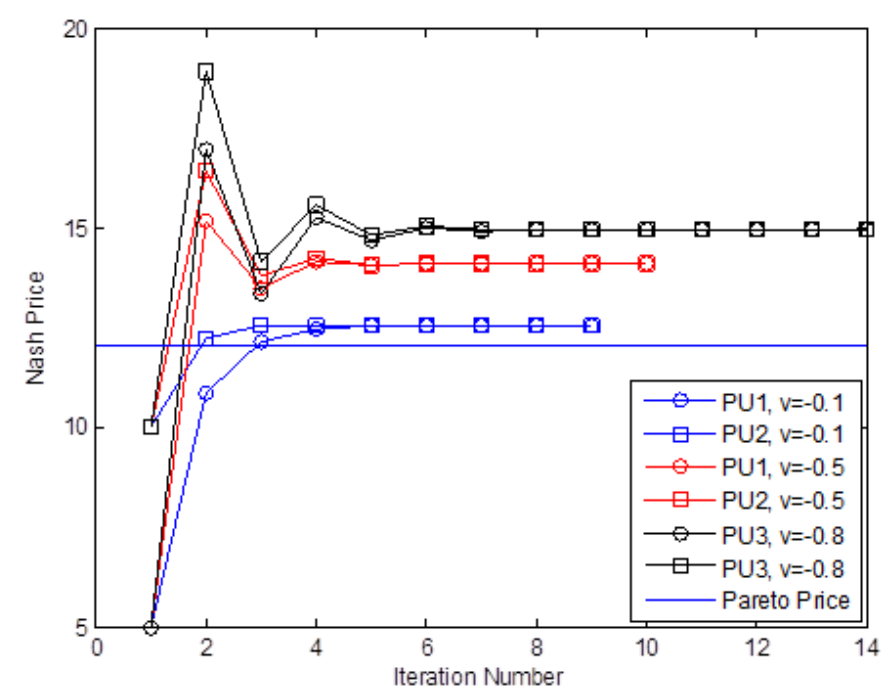

Fig. 5: The superiority of Nash price to Pareto price with the decrease of the (negative) value of $v$.

here is that, the worst case is not associated with negative substitutability parameter (which corresponds to highest PU price). This is due to the structure of the utility function used to represent the profit of the CBS. Returning to Equation (2), it can be noticed that when $v$ is negative, the third term becomes positive and add to the profit. This means that, when the penalty is put by the PU networks on spectrum switching, the profit of CBS is expected to increase! The rationale behind this attitude of the quadratic utility function at negative values of $v$ is related to what is called risk aversion. In a nutshell, the utility function in (2) takes into account not only the profit of CBS, but also the preferences of CBS. The term multiplied by $v$ indicates that the CBS would prefer to get lower (certain) profit by staying at the same spectrum instead of getting a higher (uncertain) profit by switching to another spectrum which may cause additional unexpected costs to CBS. When the penalty is increased, the CBS is more inclined to stay in the same spectrum which, according to the structure of its utility function, is preferred by the CBS.

\section{CONCLUSIONS AND RECOMMENDATIONS}

In this paper, the stability conditions for the Stackelberg algorithm for the scenario of heterogeneous cognitive network with femtocells was derived. Furthermore, the effect of the collusion game established and maintained by the PU operators on the profit of both the PU networks and the CBS has been investigated. It was found that the collusion price (Pareto price) does not represent the highest price that can be offered by the PU operators. The worst case for the CBS has been also investigated, and the simulations shows the minimum (maximum) profit achieved by the CBS is not associated with the highest price that can be offered by the PU networks. Depending on the aforementioned results, it is recommended that any deployment for a cognitive radio network, which depends on spectrum trading to provide (or to enhance) the

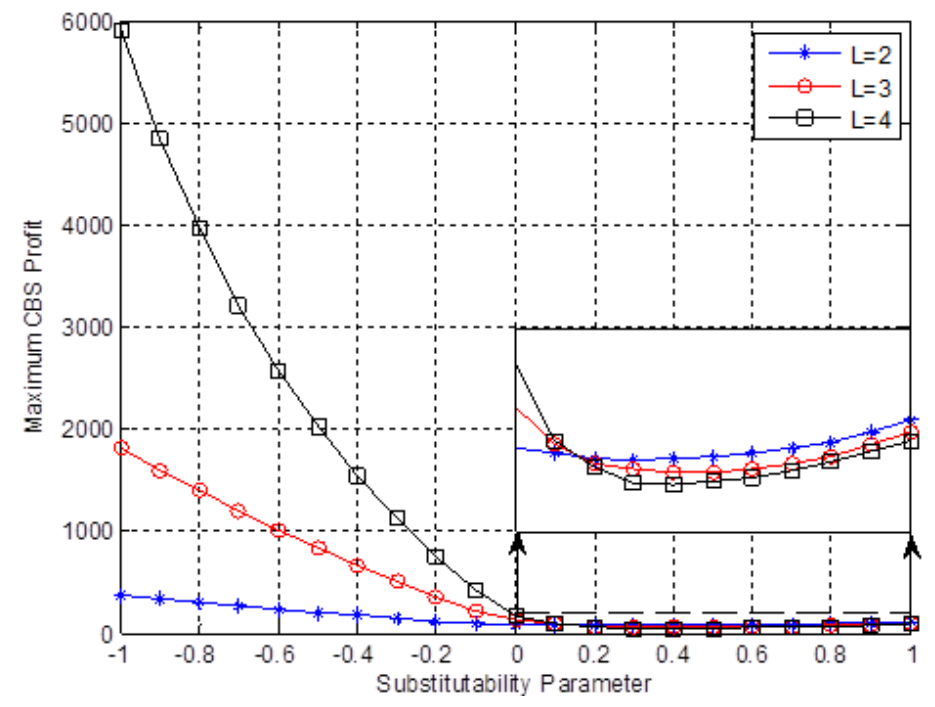

Fig. 6: The profit of CBS versus spectrum demand for different positive and negative values of $v$.

services to its users, to choose TDD deployment, because it is more flexible and reduces the number of spectrum bands required to maintain the service. Moreover, the CBS should be designed such that it can (automatically) reduce the number of spectrum bands/channels required to be purchased from the PU networks by using the available spectrum more efficiently.

\section{ACKNOWLEDGEMENT}

The authors acknowledge the support provided by King Fahd University of Petroleum and Minerals under grant number FT131009.

\section{REFERENCES}

[1] D. Grace, J. Chen, T. Jiang, and P. D. Mitchell, "Using cognitive radio to deliver 'Green' communications," Proc. of the 4th International Conference on CROWNCOM, pp. 1-6, Jun., 2009.

[2] A. Jovicic and P. Viswanath, "Cognitive radio: an information-theoretic perspective," IEEE Trans. Inf. Theory, vol. 55, no. 9, pp. 3945-3958, Aug., 2009.

[3] O. Korcak, T. Alpcan, and G. Iosifidis, "Collusion of operators in wireless spectrum markets," 2012 10th International Symposium on Modeling and Optimization in Mobile, Ad Hoc and Wireless Networks (WiOpt), pp. 3340, May, 2012.

[4] E. Kavurmacioglu, M. Alanyali, and D. Starobinski, "Competition in secondary spectrum markets: Price war or market sharing?," 2012 IEEE International Symposium on Dynamic Spectrum Access Networks (DYSPAN), Oct., 2012, pp. 440-451.

[5] D. Niyato and E. Hossain, "Competitive pricing for spectrum sharing in cognitive radio networks: dynamic game, inefficiency of Nash equilibrium, and collusion," IEEE J. Sel. Areas Commun., vol. 26, no. 1, pp. 192-202, Jan., 2008.

[6] R. Xie, F. R. Yu, H. Ji, and Y. Li, "Energy-efficient resource allocation for heterogeneous cognitive radio networks with femtocells," IEEE Trans. Wireless Commun., vol. 11, no. 11, pp. 3910-3920, Nov., 2012.

[7] Y. Saad, "Numerical methods for large eigenvalue problems," SIAM, vol. $158,1992$.

[8] P. J. Davis, "Circulant matrices," New York: Chelsea, Oct., 1994.

[9] L. C. Thomas, "Games, theory, and applications," WHILY, 1984.

[10] N. Singh and X. Vives, "Price and quantity competition in a differentiated duopoly," RAND J. Econ., pp. 546-554, 1984.

[11] A. Ivanov, "TDD-LTE and FDD-LTE: A basic comparison," ASCOM, Jan., 2012. 